\title{
The emergence of the red sequence at $z \sim 2$ seen through galaxy clustering in the UKIDSS UDS
}

\author{
William G. Hartley ${ }^{1}$, Omar Almaini ${ }^{1}$, Alice Mortlock ${ }^{1}$, Chris \\ Conselice $^{1}$ and the UDS team \\ ${ }^{1}$ School of Physics and Astronomy, University of Nottingham, University Park, Nottingham \\ NG7 2RD \\ email: will.hartley@nottingham.ac.uk
}

\begin{abstract}
We use the UKIDSS Ultra-Deep Survey, the deepest degree-scale near-infrared survey to date, to investigate the clustering of star-forming and passive galaxies to $z \sim 3.5$. Our new measurements include the first determination of the clustering for passive galaxies at $\mathrm{z}>2$, which we achieve using a cross-correlation technique. We find that passive galaxies are the most strongly clustered, typically hosted by massive dark matter halos with $\mathrm{M}_{\text {halo }}>10^{13} \mathrm{M}_{\odot}$ irrespective of redshift or stellar mass. Our findings are consistent with models in which a critical halo mass determines the transition from star-forming to passive galaxies.
\end{abstract}

Keywords. Galaxies, Evolution, Large-scale structure, etc.

\section{Introduction}

One of the most fundamental observational results in extragalactic astrophysics is the bimodal form of the galaxy population. Galaxies with spheroidal morphologies tend to have very low star-formation rates (hereafter, 'passive') and are the dominant population at high stellar masses. The more abundant disky, star-forming objects, meanwhile, typically have lower stellar mass. However, the origin of this bimodality remains unclear. A gas-rich merger or the collapse of an unstable disk is thought to result in very vigorous star-formation, possibly triggering a quasar phase. Either of these could have sufficient energy to expel the remaining gas from the galaxy, or heat it sufficiently to prevent further star formation (Silk \& Rees 1998, Fabian 1999). Without cold gas present, the galaxy can no longer form stars and it is observed as a passive object.

Recent theoretical models, however, suggest an alternative mechanism by which galaxies end their star formation. In the so called 'hot halo' model, a high mass dark matter halo can sustain a reservoir of hot gas. New gas infall is shock heated as it encounters this gas halo and is therefore not immediately available for star formation. Henceforth, only a modest amount of energy input, possibly from radio AGN feedback, is required to maintain a high gas temperature. In this way, galaxies within high mass halos become starved of star-forming gas with the result that ongoing star-formation rates become negligible. A clear consequence of the hot halo model is that there should be a characteristic halo mass scale, above which the relative fraction of passive objects (at fixed stellar mass) increases sharply. Current models suggest that this halo mass scale is a few $\times 10^{12} \mathrm{M}_{\odot}$ and becomes maximally efficient at $\mathrm{M}_{\text {halo }}>10^{13} \mathrm{M}_{\odot}$ (Croton et al. 2006, Cen 2011).

Estimates of the typical host dark matter halo of a galaxy sample can be achieved through their clustering statistics. In order to use the large-scale structure to estimate halo masses at $z>1$ we require selection at near-infrared wavelengths. A large contiguous 
field is clearly necessary to measure the bias at large scales $(\mathrm{r} \sim$ few Mpc) and find rare objects at high redshift.

Our sample is drawn from the K-band image of the UKIRT Infrared Deep Sky Survey (UKIDSS, Lawrence et al. 2007), Ultra-Deep Survey† (UDS) data release 8 and matched multi-wavelength photometry. The UDS covers 0.77 square degrees in $\mathrm{J}, \mathrm{H}$ and $\mathrm{K}$-bands, reaching depths of $\mathrm{J}=24.9$, $\mathrm{H}=24.2$ and $\mathrm{K}=24.6$ (AB magnitudes, estimated from the RMS between $2^{\prime \prime}$ apertures).

In addition to the three UKIDSS bands, the field is covered by comparable data from CFHT Megacam u-band, optical Subaru Suprime-cam data (B, V, R, i' and z'-bands) and Spitzer IRAC channels 1 and 2 . Finally, we make use of existing $24 \mu \mathrm{m}, \mathrm{X}$-ray and radio data to remove obvious AGN.

Photometric redshift probability distributions were computed for each source with dispersion of $\Delta z /(1+z) \sim 0.031$ after excluding outliers $(\Delta z /(1+z)>0.15,<4 \%$ of objects $)$. The stellar masses and rest-frame luminosities used in this work are measured using a multicolour stellar population fitting technique. We fit the 11 photometric bands to a large grid of synthetic spectral energy distributions (SEDs) constructed from the stellar population models of Bruzual \& Charlot (2003), assuming a Chabrier initial mass function.

Our separation of passive and star-forming galaxies is based initially on the $\mathrm{U}, \mathrm{V}$ and $\mathrm{J}$ Bessel band rest-frame luminosities. Objects with passive UVJ colours but SSFR $>10^{-8} \mathrm{yr}^{-1}$ derived from the template fits were re-assigned to the star-forming sample. Furthermore, the $24 \mu \mathrm{m}$ data were used to identify objects that harbour dust-enshrouded star-formation by a nearest match to the K-band catalogue within $6^{\prime \prime}$.

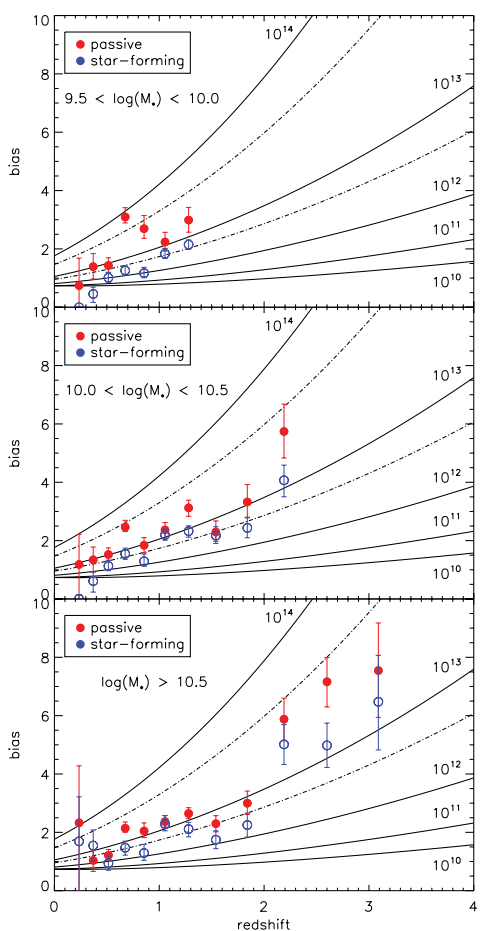

Figure 1. Galaxy bias inferred from our cross-correlation analysis against redshift for passive and star-forming samples. Filled red points represent passive samples while the open blue points are for our star-forming samples. The stellar mass range of galaxies in each panel is indicated in the upper left. Solid and dot-dashed lines show the bias for dark matter halos of various masses (in solar masses, as labelled). The dot-dashed lines are $5 \times 10^{13} \mathrm{M}_{\odot}$ and $5 \times 10^{12} \mathrm{M}_{\odot}$, upper and lower respectively.

\section{Clustering measurements}

We estimate the host dark matter halo masses of passive and star-forming sub-samples as a function of their stellar mass and redshift in intervals of $500 \mathrm{Mpc}$ across $0.3<z<$ 3.36. Using cross-correlation statistics, we can cross a small sample for which we want to know the bias (e.g. a passive sample at high redshift) with a full mass-limited tracer population and achieve much smaller statistical uncertainties on their bias, $b_{2}$, than we would through an auto-correlation analysis. The bias can be recovered as follows: 


$$
b_{2}=\frac{b_{12}^{2}}{b_{1}} \text {, }
$$

where $b_{1}$ is the bias computed from the auto-correlation function of the tracer population and $b_{12}^{2}$ is the bias measured from the cross-correlation function. In order to find $b_{12}^{2}$ we first compute the cross-correlation function using the estimator of Landy \& Szalay (1993)

We then fit a single bias parameter to the galaxy clustering measurements at large scales using the non-linear dark matter power spectrum routine of Smith et al. (2003),

$$
w_{o b s}(\theta)=b_{12}^{2} \times w_{d m}(\theta)
$$

minimising the $\chi^{2}$. Once we have computed the bias of a sample, $b_{2}$, we obtain a typical host halo mass using the models of Mo \& White (2002) (and references therein).

In Figure 1 we display the bias determined from our cross-correlation method as a function of redshift for passive and star-forming objects, with three panels representing the three bins in stellar mass. Red points correspond to our passive galaxy sample, while blue points represent the starforming objects. Any subsample in which the lower mass limit falls below the $95 \%$ mass completeness limit is not considered.

From Figure 1 a number of trends are apparent. Firstly, passive galaxies are, on average, clearly more strongly clustered than their star-forming counterparts at similar stellar mass. This result is most apparent at lower stellar masses $(\mathrm{M}<$ $\left.10^{10} \mathrm{M}_{\odot}\right)$ and for subsamples at $z<1$.

A second feature of these results is the mass dependence within samples. The least massive of the passive galaxies appear to be the most strongly clustered (and therefore in the most massive dark matter halos). The bias typically decreases as the masses of the subsamples increase. Despite this mass dependence, our passive galaxy samples appear to have consistently high parent halo masses with typical masses $M_{\text {halo }} \geqslant 10^{13} \mathrm{M}_{\odot}$ or higher.

For star-forming sub-samples, galaxies of different stellar masses but similar redshifts do not show any clear trends. The measurements are consistent with star-forming galaxies being typically hosted by similar mass halos irrespective of stellar mass. This absence of a clear correlation for starforming galaxies was identified in the luminosityselected subsamples in Hartley et al. (2010). Similarly, the downsizing-like behaviour we found in Hartley et al. is also present in this work, with subsamples of equal stellar mass typically hosted by less massive halos at low redshift.

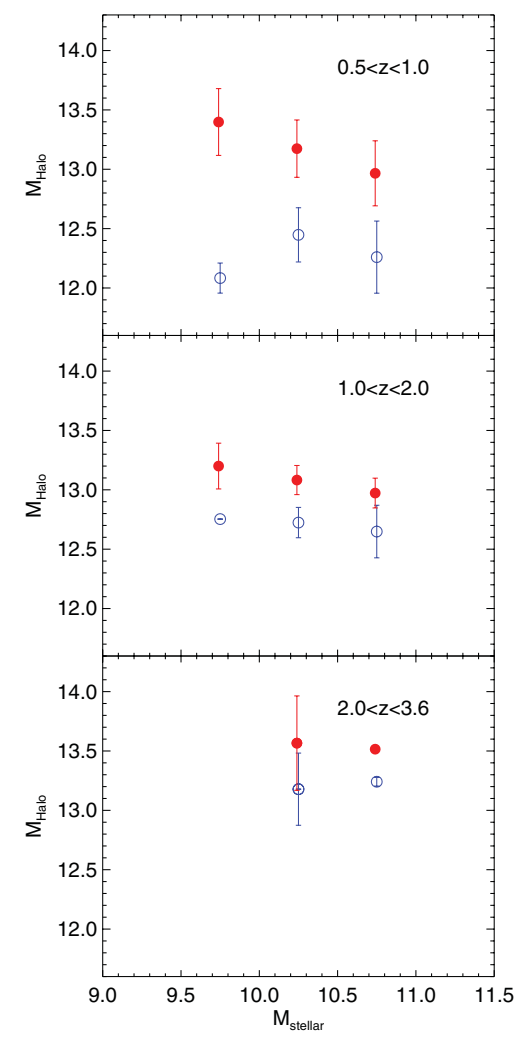

Figure 2. Average halo masses implied by our bias measurements in three redshift intervals (as labelled). The data represent mean halo masses for samples of fixed stellar mass, while the uncertainties are the standard error on the mean. As in previous Figures, filled red points represent passive galaxy samples and open blue points are for star-forming objects. 
The raw measurements presented in Figure 1 are difficult to fully digest. We therefore condense our results into a more intuitive set of plots in Figure 2. We plot the mean halo mass implied by our clustering results as a function of stellar mass for passive and star-forming subsamples in three redshift ranges: $0.5<z<1.0,1.0<z<2.0$ and $2.0<z<3.36$. Each point in Figure 1 is converted to a typical halo mass, based on the measured bias, and these are then averaged over the indicated redshift intervals. The uncertainties on the data are the standard error on the mean.

The behaviour described above can be seen more clearly in Figure 2: the strong clustering of low-mass passive samples, the lack of a stellar mass dependence on halo mass for star-forming galaxies and the 'downsizing' of halo masses with redshift are all apparent. The averaged halo masses of all passive galaxy samples are greater than or equal to $10^{13} \mathrm{M}_{\odot}$ and within each redshift range the lowest-mass galaxies are found in the most massive halos. This result is clearest at low redshifts, indicating a build up of relatively low mass passive objects in high mass halos. Although there is no clear stellar mass dependence on halo mass for star-forming galaxies, the downsizing-like behaviour is clearly apparent. Typical halo masses in our lowest redshift interval are $10^{12-12.5} \mathrm{M}_{\odot}$, while they exceed $10^{13} \mathrm{M}_{\odot}$ at the highest redshifts.

Taken at face value, the average parent halo masses for passive galaxy subsamples shown in Figure 2 are in agreement with a hot halo model and consistent with a limiting mass scale for passivity. Passive galaxies of all masses are typically hosted by halos of $\mathrm{M}_{\text {halo }} \geqslant 10^{13} \mathrm{M}_{\odot}$ or greater. The typical host halo masses for star-forming galaxies show no consistent stellar mass dependence. However, at $z>2$ our star-forming samples are typically also found in halos with mass, $M_{\text {halo }} \geqslant 10^{13} \mathrm{M}_{\odot}$. It is therefore possible that the efficiency with which a hot halo can shut-off star-formation evolves with redshift. We will conduct an interpretation of our results in the context of the halo occupation framework in future work.

\section{References}

Bruzual, G. \& Charlot, S. 2003, MNRAS, 344, 1000

Cen, R. 2011, ApJ, 741, 99

Croton, D. J., et al. 2006, MNRAS, 365, 11

Fabian, A. C. 1999, MNRAS, 308, L39

Hartley, W. G., et al. 2010, MNRAS, 407, 1212

Landy, S. D. \& Szalay, A. S. 1993, ApJ, 412, 64

Lawrence, A., et al. 2007, MNRAS, 379, 1599

Mo, H. J. \& White, S. D. M. 2002, MNRAS, 336, 112

Silk, J. \& Rees, M. J. 1998, A\&GA, 331, L1

Smith, R. E., et al. 2003, MNRAS, 341, 1311 